SHS Web of Conferences 20, 01023 (2015)

DOI: $10.1051 /$ shsconf $/ 20152001023$

(C) Owned by the authors, published by EDP Sciences, 2015

\title{
Recueillir des données auprès d'élèves avec troubles du spectre autistique en collège
}

\section{Collecting data from students with autistic spectrum disorders in secondary school}

\author{
Sabine Zorn ${ }^{1, a}$, Minna Puustinen ${ }^{1}$ \\ ${ }^{1}$ EA 7287 Grhapes, INS HEA, 58-60 avenue des Landes, 92150 Suresnes, France
}

\begin{abstract}
Résumé. Cet article a pour objectif de présenter les étapes et méthodes d'un recueil de données auprès de collégiens avec troubles du spectre autistique (TSA) inscrits en Ulis (Unité localisée pour l'inclusion scolaire) collège et suivant une partie de leurs enseignements en classe ordinaire. L'étude en question vise à mieux comprendre la nature et le déroulement des interactions sociales en classe entre des collégiens avec TSA et leurs enseignants. La première partie de l'article fournit un certain nombre d'éléments contextuels (loi de 2005, modalités de scolarisation des élèves avec TSA, recherches existantes sur les spécificités sociocognitives et la scolarisation des élèves avec TSA). Notre démarche de recueil de données, décrite dans la deuxième partie de l'article, tient compte de ces éléments contextuels ainsi que des méthodes utilisées par d'autres chercheurs dans ce domaine. Une analyse de toutes ces données a abouti à un dispositif composé de quatre étapes préliminaires (constitution de l'échantillon, recueil des consentements, choix et préparation des méthodes de recueil de données, vérification de la conformité du projet avec la loi relative à l'informatique, aux fichiers et aux libertés) et de plusieurs méthodes de recueil de données impliquant quatre sources d'information (observation filmée, entretien, examen des dossiers, test standardisé).
\end{abstract}

\begin{abstract}
The aim of this article is to present the steps and methods of data collection focusing on secondary school students with autism spectrum disorders (ASD) who attend some classes in a resource classroom, and some in an ordinary classroom. The study in question aims at better understanding the nature of social interactions taking place in the classroom between these students and their teachers. The first part of the paper provides some contextual information (law of February 2005, existing organization of schooling for secondary school students with ASD, research concerning socio-cognitive issues and education of students with ASD). The data collection methodology is described in the second part of the paper and takes into account the contextual elements as well as the methodological choices made by other researchers in this domain. Analysis of all these elements resulted in a plan composed of four preliminary steps (constituting the research sample, collecting consent forms, choosing and preparing data collection methods, verifying the compliance of the research project with the law on information technology, data files and civil liberties) and several data collection methods involving four information sources (filmed observations in natural contexts, interviews, review of school and medical records, a standardized test).
\end{abstract}

\section{Introduction}

Cet article a pour objectif de présenter les étapes et méthodes d'un recueil de données qui s'est déroulé en milieu scolaire auprès d'adolescents avec troubles du spectre autistiques (TSA). Le recueil

\footnotetext{
a Auteur de correspondance : sabine.zorn@inshea.fr
}

This is an Open Access article distributed under the terms of the Creative Commons Attribution License 4.0, which permits unrestricted use, distribution, and reproduction in any medium, provided the original work is properly cited. 
dont il est question a été réalisé dans le cadre d'une recherche de doctorat en psychologie. L'étude menée vise à mieux comprendre la nature et le déroulement des interactions sociales en classe entre des collégiens avec TSA et leurs enseignants. Notre réflexion s'inscrit dans un cadre théorique qui considère que l'analyse des interactions sociales est un moyen privilégié de compréhension des phénomènes scolaires d'apprentissage, d'éducation et plus généralement d'intégration sociale et culturelle (Coulon, 1993 [1]). Compte tenu des spécificités liées à la scolarisation de ces élèves, nous commencerons par un rappel des éléments contextuels. Nous nous intéresserons ainsi d'abord aux modalités actuelles de scolarisation des jeunes avec TSA en France puis analyserons leurs particularités sociocognitives et nous nous familiariserons avec les travaux existants portant sur leur scolarisation. Par la suite, nous présenterons les choix méthodologiques qui ont guidés notre recueil de données auprès de cette population.

\section{La scolarisation des élèves avec handicap en France}

Comme la plupart des travaux actuels portant sur les élèves avec handicap en France, notre travail s'inscrit dans le contexte de la loi du 11 février 2005 affirmant un droit à la scolarisation des enfants et adolescents porteurs de handicap. Cette loi remplace celle de 1975 qui avait juridiquement établi le principe d'éducabilité en posant le droit à une éducation pour tout enfant handicapé. Elle va plus loin en affirmant que tout enfant avec handicap est de droit un élève. Dans ce contexte, le nombre d'élèves scolarisés en milieu ordinaire en France a considérablement progressé. Pour ce qui est de l'enseignement secondaire, les effectifs sont passés de 28789 élèves avec handicap scolarisés dans le milieu ordinaire à 97560 élèves en sept ans (2006-2013) soit une évolution de 238,9\% (SGMAP, 2013 [2]). Le nombre d'élèves avec TSA (premier et second degrés confondus) scolarisés dans le milieu ordinaire, quant à lui, est passé de 12500 élèves à 20375 élèves en quatre ans (2008-2012; enquêtes 3 et 12, DGESCO [3]), ce qui représente une évolution de 61,3\%. Or sur ces 20375 élèves, seuls $19 \%$ sont scolarisés en collège et 3,5\% en lycée général ou professionnel. Dans ce contexte, il semble important de faire évoluer cette situation et d'accroître le nombre d'élèves avec TSA scolarisés en milieu ordinaire dans l'enseignement secondaire. Notre étude s'inscrit dans cette perspective.

\subsection{Les modalités de scolarisation des adolescents avec TSA}

Plusieurs modalités de scolarisation existent pour les adolescents avec handicap en France. Elles ont considérablement évolué depuis la loi de 2005 et en ce qui concerne les élèves avec TSA. Elles sont présentes dans différents secteurs d'activité : le secteur sanitaire, le secteur médico-social et le secteur éducatif.

Le jeune avec TSA peut être inscrit dans un établissement du secteur sanitaire, c'est-à-dire dans un établissement géré par la fonction hospitalière. Dans ces établissements qui accueillent des jeunes durant la journée (hôpital de jour), un enseignant peut être mis à disposition par l'Education nationale pour assurer des heures d'enseignement à l'ensemble ou une partie des jeunes. Dans ce cas, l'établissement dispose d'une Unité d'enseignement (UE) dans laquelle l'enseignant exerce. En fonction du projet éducatif du jeune avec TSA, ce dernier peut bénéficier de quelques heures de scolarisation avec l'enseignant. Toutefois, ce temps est limité de $1 \mathrm{~h}$ à $5 \mathrm{~h}$ environ d'enseignement par semaine.

D'autre part, le jeune avec TSA peut être inscrit dans un établissement du secteur médico-social qui se nomme un Institut médico-éducatif (IME). Dans ces établissements qui accueillent des jeunes durant la journée (et parfois en internat), une UE peut aussi exister. Dans ce cas, un enseignant est également présent pour assurer des heures de scolarité à l'ensemble ou une partie des jeunes, en fonction des projets éducatifs de ces derniers. Ainsi, le jeune avec TSA peut bénéficier d'un temps de scolarisation mais il reste également limité (de $1 \mathrm{~h}$ à $5 \mathrm{~h}$ environ par semaine).

Dans les deux modalités de scolarisation ci-dessus, le choix peut être fait de faire suivre au jeune ses heures d'enseignement dans un établissement ordinaire du système éducatif. Dans ce cas, le jeune suit en général ces enseignements en Ulis (Unité localisée pour l'inclusion scolaire) école (ex-Clis 
dans le premier degré), collège ou lycée (dans le second degré). Les heures de scolarisation sont aussi limitées mais peuvent atteindre jusqu'à une quinzaine d'heures par semaine, soit deux journées et demie. Le jeune peut être accompagné ponctuellement (quelques heures) par un personnel (éducateur, moniteur-éducateur, etc.) de son établissement de rattachement.

Enfin, le jeune peut être inscrit dans un établissement du système éducatif, c'est-à-dire à l'école, en collège ou en lycée, et dans ce cas, il a deux possibilités (en fonction de ses besoins éducatifs particuliers) :

- $\quad$ suivre l'ensemble de ses enseignements en classe ordinaire, autrement dit en inclusion totale, avec si nécessaire un accompagnement humain grâce à la présence d'un AESH (accompagnant des élèves en situation de handicap) ou d'un auxiliaire de vie scolaire (AVS) individuel ou collectif (mutualisé). Cet accompagnant peut être présent sur l'ensemble du temps de scolarisation ou à temps partiel ;

- $\quad$ suivre une partie de ses enseignements en Ulis et une autre partie en classe ordinaire (classe d'inclusion). De la même manière que ci-dessus, un AESH ou un AVS individuel ou collectif peut accompagner le jeune durant ces deux situations d'apprentissage.

Une troisième possibilité de scolarisation dans le système éducatif existe mais elle est a priori moins fréquente ${ }^{\mathrm{b}}$, il s'agit de la scolarisation en Section d'enseignement générale et professionnelle adaptée (Segpa). Le jeune avec TSA suit des enseignements avec des élèves en difficultés scolaires graves et durables dans une classe à effectif réduit (16 élèves environ par section). La formation en Segpa conjugue des enseignements généraux et des activités pratiques préparant à l'accès à une formation professionnelle. Les enseignements mettant en œuvre des activités pratiques s'appuient sur cinq champs professionnels : habitat, hygiène-alimentation services, espace rural et environnement, vente-distribution-magasinage, production industrielle.

Le choix de l'une ou l'autre des modalités de scolarisation se fait en fonction des besoins éducatifs particuliers (BEP) du jeune. Ceux-ci sont étudiés par la Commission des droits et de l'autonomie des personnes handicapées (CDAPH) de la Maison départementale des personnes handicapées (MDPH) à laquelle le jeune doit être inscrit pour bénéficier d'un projet personnalisé de scolarisation (PPS). Son affectation est ensuite conditionnée par l'évaluation de sa situation par la commission et par le nombre de places disponibles dans les établissements existants.

Dans cet article, nous nous intéressons exclusivement aux adolescents avec TSA scolarisés en collège et suivant une partie de leurs enseignements en Ulis collège et une autre partie en classe ordinaire. En effet, 42,3\% des élèves avec TSA scolarisés en collège le sont en Ulis collège (DGESCO, 2011-2012 [3]). Il s'agit d'élèves pour qui l'inclusion totale en classe ordinaire n'est pas encore possible mais pour qui il est souhaitable d'accroître autant que possible les temps de scolarisation en milieu ordinaire. Compte tenu de notre contexte de travail (la loi du 11 février 2005 favorisant l'inclusion scolaire), ce choix nous a semblé particulièrement pertinent. De ce fait, en comparant le comportement des mêmes élèves dans deux situations d'apprentissage différentes (la classe ordinaire et le dispositif Ulis qui se situe à mi-chemin entre la classe ordinaire et la classe spécialisée, cf. chapitre 2.2), nous pourrons sans doute mieux comprendre l'impact des particularités du fonctionnement autistique sur les apprentissages et envisager des stratégies d'accompagnement à la scolarisation de ces élèves en vue d'une meilleure inclusion.

\subsection{La scolarité en Ulis collège}

La scolarité d'un élève avec TSA en Ulis collège s'organise autour des deux principes administratifs fondamentaux suivants :

\footnotetext{
${ }^{\mathrm{b}}$ Les Segpa ont été conçues initialement pour des élèves ayant des difficultés scolaires graves et durables. Or, la CDAPH et la Commission départementale d'orientation vers les enseignements adaptés du second degré (CDOEA) peuvent malgré tout orienter ponctuellement les élèves avec handicap dans ces sections.
} 
- l'inscription administrative de l'élève dans une classe ordinaire correspondant à sa classe d'âge (par exemple, si l'élève à 12 ans, il est inscrit en classe de $6^{\circ}$, s'il a 13 ans, il est inscrit en classe de $5^{\circ}$, etc.), cette classe étant alors sa classe d'inclusion;

- l'inscription de l'élève dans le dispositif Ulis.

Le collégien a un emploi du temps spécifique dans lequel sont répartis les différents temps d'enseignement. Cet emploi du temps est construit par l'enseignant de l'Ulis en collaboration avec les enseignants de la classe ordinaire et le chef d'établissement. Il est formalisé par la rédaction du PPS de l'élève. Ce document mentionne également les objectifs pédagogiques et sociaux sous-jacents.

Les temps d'enseignement en Ulis et en classe ordinaire ont chacun leurs caractéristiques :

- Les temps d'enseignement en Ulis sont dispensés par un enseignant du premier ou du second degré spécialisé dans les troubles des fonctions cognitives (TFC). Cette spécialisation s'obtient par la validation du Certificat d'aptitude professionnelle pour les aides spécialisées, les enseignements adaptés et la scolarisation des élèves en situation de handicap (Capa-SH) pour les troubles des fonctions cognitives (option D) pour les enseignants du premier degré, et du Certificat complémentaire pour l'adaptation scolaire et la scolarisation des élèves handicapés (2CA-SH) option D pour les enseignants du second degré. Un AESH ou un AVS collectif et d'autres élèves porteurs de troubles des fonctions cognitives sont également présents dans le dispositif. Ils sont entre 10 à 12 élèves, ont entre 12 et 16 ans et des niveaux scolaires variés allant du milieu du cycle $2(\mathrm{CP})$ à la fin du collège. Ils ont tous des troubles des fonctions cognitives (dyslexie, déficience intellectuelle, etc.), et dans certaines Ulis, tous les élèves ont des TSA.

- Les temps d'enseignement en classe ordinaire sont dispensés par un enseignant du second degré. Dans la classe ordinaire, les effectifs varient souvent de 25 à 30 élèves environ. Parfois, plusieurs élèves de l'Ulis sont inclus en même temps dans la même classe. Un AESH ou un AVS (collectif ou individuel) peut également accompagner l'élève.

\section{Les élèves avec TSA}

Les recherches sur les TSA sont nombreuses et touchent des domaines variés comme l'étiologie, le développement ou les thérapies. En ce qui concerne l'éducation en milieu ordinaire et plus particulièrement les enseignements scolaires, il existe moins de travaux. Ceci s'explique sans doute en partie par le manque de recul vis-à-vis de la loi de 2005, peut-être aussi par la complexité des situations à analyser et la nécessité de travailler avec plusieurs cadres théoriques (sciences de l'éducation, psychologie, etc.). Nous présentons dans la partie qui suit les particularités sociocognitives de ces apprenants et les avancées de la recherche dans le domaine de leur scolarisation.

\subsection{La définition des TSA}

Les troubles du spectre autistique se définissent par 1) des troubles de la communication sociale et 2) des intérêts et comportements restreints (APA, 2013 [4]). Les causes d'apparition de ces troubles sont encore inconnues et, même si plusieurs hypothèses étiologiques cohabitent, aucun consensus scientifique n'existe à ce jour. De ce fait, un diagnostic de TSA se base aujourd'hui exclusivement sur des symptômes comportementaux observables. Par ailleurs, de grandes variabilités interindividuelles existent parmi les personnes obtenant un diagnostic de TSA. En effet, le spectre des troubles est vaste et englobe autant des personnes ayant un faible niveau de fonctionnement socio-communicatif avec une déficience intellectuelle que des personnes ayant de très bonnes capacités intellectuelles. De ce fait, les généralisations sur l'ensemble de la population des personnes avec TSA sont difficiles voire impossibles à réaliser. Il est préférable de préciser les caractéristiques de la population étudiée au sein du spectre des troubles. La distinction régulièrement retrouvée dans les recherches sur les TSA est 
l'absence ou la présence de déficience intellectuelle, ce qui n'est pas toujours suffisant vue la complexité du tableau clinique des personnes avec TSA.

\subsection{Les spécificités sociales des élèves avec TSA}

On sait aujourd'hui que "les difficultés observées dans le fonctionnement socio-communicatif des enfants avec TSA s'étendent à tous les canaux d'échange (visuels, gestuels, vocaux et verbaux) dès les composantes les plus élémentaires de ces habiletés (accordages expressifs et sensori-moteurs interpersonnels et coordination intrapersonnelle des moyens d'échanges) » (Plumet, 2014, p. 107 [5]). Cependant, ces anomalies ne sont pas à considérer simplement au niveau quantitatif (faible durée des échanges, par exemple) mais aussi au niveau qualitatif (formes d'échanges peu conventionnelles, registre limité des fonctions communicatives, etc.). Par exemple, l'idée d'évitement des contacts sociaux véhiculée couramment sur ces personnes relève d'une réalité bien plus complexe. Des études sur la motivation sociale, par exemple, apportent des éléments de nuance. A partir de la micro-analyse d'interactions filmées entre enfants avec TSA, leur mère ou un adulte psychologue, une étude montre que le nombre des signaux émis par l'enfant envers autrui ne diffère pas significativement des populations contrôles (Dawson et al., 1990 ; Wetherby \& Prutting, 1984 [6-7]). Cependant ce qui diffère nettement dans les échanges, ce sont les coordinations entre signaux, c'est-à-dire que les regards ou sourires de l'enfant apparaissent peu en réponse à ceux de l'adulte et que l'enfant combine mal ces signaux entre eux. De plus, non seulement les signaux interprétés comme sociaux sont fréquemment émis par les enfants avec TSA, mais il est aussi possible que certaines de leurs initiatives communicatives échappent à l'interlocuteur car elles prennent des formes inhabituelles, ne respectant pas les codes communs d'interaction (Feldstein et al., 1982 [8]).

Ainsi, il existe un certain nombre de fonctions a priori déficitaires chez les enfants et adolescents avec TSA mais il convient de le comprendre au-delà de la simple présence ou absence de comportement :

- La capacité de l'attention conjointe qui représente le fait de partager avec autrui un événement, d'attirer et de maintenir son attention vers un objet ou une personne dans le but d'obtenir un regard conjoint, avec conscience du partage d'attention (Bruner, 1983 [9]), est atypique chez les enfants avec TSA. Cette capacité qui apparaît normalement vers 9-10 mois se met difficilement en place sans rééducation spécifique chez ces enfants. Ainsi, on note des difficultés à utiliser et comprendre le pointage gestuel et, l'utilisation du corps de l'autre comme prolongement de son corps. De plus, des enfants avec autisme mis en présence d'objets, qui suscitent leur intérêt, et d'un adulte, manifestent très peu l'usage des regards interactifs (voir Bruinsma et al., 2004 [10], pour une revue de question).

- La capacité à imiter qui se trouve présente aussi très tôt voire dès la naissance (par exemple, Meltzoff \& Moore, 1977 ; Piaget, 1945 [11-12]) a longtemps été jugée absente chez les enfants avec TSA. Aujourd'hui, grâce, entre autres, aux travaux de Nadel (1986 [13]) en France sur l'imitation chez l'enfant avec TSA, on sait qu'il existe des déficits au niveau de l'imitation spontanée et différée mais qu'il existe aussi de réelles capacités d'imitation immédiate en situation d'interactions, surtout dans un répertoire moteur non symbolique (Nadel, 2011 [14]).

- La capacité à construire une théorie de l'esprit a été en premier lieu étudiée par Baron-Cohen et al. (1985 [15]). L'hypothèse d'un trouble a alors été avérée chez les personnes avec TSA qui montrent des difficultés à reconnaître/identifier pour eux-mêmes des états mentaux et à les attribuer aux autres. De ce fait, il peut être difficile pour les élèves avec TSA de prendre en compte le point de vue de l'autre, de faire preuve d'empathie et d'adaptabilité sociale.

- La capacité à communiquer leurs émotions pose aussi question chez les personnes avec TSA. En effet, ces enfants et adolescents manifestent souvent des réactions affectives excessives (grosses colères, peurs incompréhensibles), trop faibles (impression d'indifférence à la détresse ou à la joie) ou inappropriées (expressivité émotionnelle perçue comme guindée, manque de 
spontanéité ; Vermeulen, 2009 [16]). Un défaut qualitatif de l'ajustement affectif est aussi décrit au plan de la posture du corps, de l'attitude trop molle ou raidie dans les bras de la mère ainsi que peu d'harmonisation de la tonalité des vocalisations ou des verbalisations. Les observations cliniques indiquent que ces enfants peuvent manifester du plaisir à des contacts plus «rudes» comme les jeux de bousculade mais il est difficile de savoir si ce qui est recherché ici par l'enfant est un échange véritablement social ou une stimulation sensorimotrice via autrui. Ces descriptions coïncident avec le témoignage de Temple Grandin (1992 [17]), personne avec le syndrome d'Asperger, qui expliquait son besoin physique et psychologique d'être serrée très fortement pour réguler ses émotions. Elle avait ainsi fabriqué une « machine à serrer » qui lui permettait d'exercer des pressions sur son corps quand cela lui semblait nécessaire.

Ainsi, les spécificités sociales sont potentiellement nombreuses chez les élèves avec TSA en milieu scolaire. Pour autant, "être autiste ne signifie pas être indifférent » aux autres mais plutôt être différent "comme un extraterrestre échoué sans manuel d'orientation » (témoignage de Jim Sinclair, in Schopler \& Mesibov, 1992, p. 294 [18]). Dans ce contexte, plusieurs méthodes permettant de pallier les déficits sociaux des jeunes avec TSA ont été développées (voir Poirier \& Des RivièresPigeon, 2013 [19]). De plus, l'importance de la participation des élèves tout-venants à ces programmes d'aide aux interactions sociales a été, à plusieurs reprises, démontrée (Watkins et al. 2014 [20]). Par ailleurs, des recherches portant sur l'analyse des interactions sociales en classe entre les élèves avec TSA et leurs pairs, sans programme spécifique, montrent que des interactions sociales existent bien mais qu'elles nécessitent un soutien de la part des adultes. En effet, lorsque les élèves tout-venants ont pour consigne d'aider leurs camarades avec TSA (Vandromme, 1993 [21]), des stratégies d'aide et d'adaptation envers les élèves avec TSA se développent effectivement. Cependant, les interactions, surtout quand elles sont spontanées, ne sont pas toujours de nature à fournir de l'aide aux élèves avec TSA (Berzin \& Lebert-Candat, 2006 [22]) ; certains comportements des élèves toutvenants ou des élèves avec TSA viennent perturber les interactions. Dans ce contexte, Vandromme (1993 [21]) constate que les stratégies développées par les adultes sont plus adaptées aux besoins des élèves avec TSA que celles développées par les pairs. Ainsi, tout comme le préconisait Tardif (1994 [23]), Berzin et Lebert-Candat (2006 [22]) suggèrent que la formation préalable des élèves toutvenants à l'accueil des élèves avec TSA favoriserait leur inclusion sociale et scolaire.

\subsection{Les spécificités cognitives des élèves avec TSA}

Les fonctions cognitives globales telles que la mémoire, le fonctionnement sensoriel, l'activité imaginative et symbolique, les capacités en catégorisation et les fonctions exécutives s'organisent de manière particulière chez les personnes avec TSA. L'étendue des particularités cognitives des élèves avec TSA fait de ces apprenants des sujets particulièrement difficiles à évaluer et pour lesquels un bon nombre de pratiques pédagogiques sont remises en question. Voici succinctement les grands traits de leurs caractéristiques cognitives :

- En ce qui concerne les capacités mnésiques, les performances sont paradoxales : certains personnes avec TSA disposent de compétences étonnantes pour se rappeler les noms des personnes environnantes, des itinéraires, des mélodies complexes, etc. (Kanner, 1943 [24]), tandis que d'autres ont des anomalies qualitatives de mémorisation des informations (Marcaggi et al., 2010 [25]). Il semble que dans le cas des capacités mnésiques importantes, il s'agisse plus d'un signe d'une organisation cognitive atypique que d'un indice d'une intelligence supérieure. De manière générale, les auteurs rattachent plutôt les particularités d'utilisation de la mémoire à d'autres secteurs cognitifs. Par exemple, les problèmes en mémoire autobiographique ou épisodique seraient plutôt liés aux particularités du développement de la théorie de l'esprit, les problèmes de mobilisation de la mémoire de travail seraient plutôt liés à des dysfonctionnements exécutifs, etc. 
- En ce qui concerne les capacités en catégorisation, Frith et Happé (1994 [26]) proposent l'hypothèse du trouble de la "cohérence centrale ». L'enfant avec TSA aurait du mal à se détacher des niveaux de traitement localistes des informations (attachement aux détails ou à des blocs d'informations figés) et à en dégager une structure signifiante de plus haut niveau, pertinente en fonction du contexte. Certains auteurs nuancent cette théorie en disant que les difficultés de l'enfant avec TSA sont plutôt de l'ordre de la hiérarchisation des différents niveaux de traitement et du passage souple de l'un à l'autre selon les besoins de la situation (Mottron, 2004 [27]).

- En ce qui concerne l'activité symbolique des personnes avec TSA, des idées contradictoires ont été émises : d'une part que ces personnes n'avaient pas accès au symbolique et d'autre part qu'elles avaient une vie imaginative interne débordante. Comme avec la plupart des fonctions cognitives explorées chez ces personnes, les anomalies sont plus à chercher du côté des différences qualitatives que d'une incapacité à construire des symboles. Preissler (2008 [28]), étudiant une gamme variée de symboles (graphiques, verbaux en mode oral ou écrit, etc.) construite par les individus avec TSA, montre que la relation symbole-référent est bien existante mais qu'elle est difficilement communicable et partageable avec autrui. Ainsi, comme en témoigne Tammet (2007 [29]) et son propre code symbolique basé sur les couleurs et les nombres, la fonction symbolique existe bien chez les personnes avec TSA mais elle ne correspond pas aux systèmes culturels collectifs.

- En ce qui concerne les fonctions exécutives, c'est-à-dire l'ensemble des processus mentaux supérieurs permettant d'organiser une action (attention, intention, anticipation, planification, inhibition d'automatismes, mémoire de travail, contrôle de la performance et feedback...), on retrouve chez l'ensemble des personnes avec TSA des défauts d'utilisation. On remarque des défauts de flexibilité cognitive (Ozonoff \& McEvoy, 1994 [30]) de même que des difficultés à planifier l'action. Ceci pourrait expliquer certains de leurs comportements de persévération, leur manque d'initiative et leur difficulté à maintenir et réguler leur attention.

- Enfin, en ce qui concerne le fonctionnement sensoriel, on constate un comportement particulier par rapport aux sons (par exemple, pas de réaction au claquement d'une porte mais vive réaction au papier qui se froisse), aux lumières (par exemple, attention soutenue à la vue d'une ampoule allumée mais problème d'attention par ailleurs), aux odeurs et aux contacts tactiles (par exemple, préférence pour les parties rugueuses d'un objet plutôt que pour les caresses avec une peluche). Les examens pratiqués pour vérifier les systèmes sensoriels ne détectent en général aucune anomalie. Plusieurs travaux expérimentaux ont permis de confirmer les réactions paradoxales des enfants avec TSA aux stimulations dans les différentes modalités sensorielles. Les auteurs parlent d'hyper-réactions ou d'hypo-réactions par rapport aux sujets normaux.

\subsection{Des stratégies d'apprentissage spécifiques}

Pour pallier les particularités des apprenants avec TSA et anticiper les difficultés qu'ils pourraient rencontrer en milieu scolaire, des méthodes spécifiques d'apprentissage (TEACCH, $\mathrm{ABA}^{\mathrm{c}}$ ) ont été utilisées outre-Atlantique (Leaf \& Eachin, 2006 ; Schopler \& Mesibov, 1986 [31-32]) et en Europe (Beaugerie-Perrot \& Lelord, 1991; Jordan \& Powell, 1997 ; Peeters, 1988 ; Wing, 1985 [33-36]) depuis plus de trente ans. Ces méthodes proposent de combiner des aides au développement des compétences interactives de base (attention, perception, intention, imitation, etc.) et des aides aux compétences plus complexes (organisation, gestion des émotions, maintien des interactions, etc.). L'évaluation de ces méthodes a fait l'objet en France d'un rapport de la Haute Autorité de Santé (HAS

c TEACCH : Treatment and education of autistic and related communication handicapped children. ABA : Applied behavior analysis. 
[37]) en 2012. Malheureusement, peu de travaux se sont intéressés à leur usage en situation scolaire ordinaire alors que la loi de 2005 met en avant l'intérêt de la scolarisation en milieu ordinaire pour tous les enfants et adolescents porteurs de handicap.

Depuis une dizaine d'années, des recherches se développent pour étudier les pratiques enseignantes adaptées aux élèves avec TSA en milieu inclusif (pour des revues de question, voir Corneau et al., 2014 ; Crosland \& Dunlap, 2012 ; Harrower \& Dunlap 2001 [38-40]). Ces auteurs recensent les études portant sur les stratégies dites " efficaces » pour les élèves avec TSA en contexte inclusif (par exemple : les stratégies basées sur les antécédents, les stratégies d'autogestion, la médiation par les pairs, l'entraînement aux habilités sociales). Dans le même temps, ils pointent des problèmes méthodologiques majeurs aux études recensées : la petite taille des échantillons (beaucoup d'études de cas), la difficile évaluation des stratégies (comment évaluer l'efficacité d'une stratégie ?), la variabilité des profils autistiques (le spectre autistique est très vaste) ainsi que le manque de recherches menées dans l'enseignement secondaire. Dans un domaine proche, Koegel et al. (2012 [41]) recensent les interventions pédagogiques et éducatives en milieu inclusif qui permettent de réduire les troubles du comportement, d'augmenter les compétences de communication et les interactions sociales des élèves avec TSA. Ces auteurs montrent les difficultés d'implantation de ces interventions dans le système scolaire ordinaire. Ils recommandent de recueillir des informations via différentes sources afin d'évaluer précisément les situations scolaires en vue de l'implantation d'un programme spécifique d'aide à l'inclusion des élèves avec TSA.

\subsection{Les attitudes des enseignants envers les élèves avec TSA}

Prenant en considération les recherches portant sur les stratégies adaptées aux apprenants avec TSA et les différents textes de loi sur la scolarisation des élèves avec handicap en fonction des pays, une partie de la recherche sur la scolarisation et des élèves avec TSA s'intéresse de près à l'attitude des enseignants vis-à-vis des élèves avec TSA (Chung et al., 2015; Humphrey \& Symes, 2013 ; McGregor \& Campbell, 2001 ; Roberston et al., 2003 ; Soto et al., 2012 [42-46]). Plus précisément, ces études montrent le rôle clé de l'enseignant dans l'inclusion scolaire des élèves avec TSA. Les facteurs relatifs aux enseignants évoqués sont leur attitude, leur formation, le contexte scolaire dans lequel ils enseignent, leur conception des TSA et de l'inclusion scolaire en général. Les résultats concernant l'attitude des enseignants vis-à-vis de l'inclusion des élèves avec TSA sont partagés car en fonction du type de recrutement des participants, de la zone géographique où se déroule l'étude et des questionnaires utilisés, les attitudes des enseignants peuvent varier (Segall \& Campbell, 2012 [47]). Dans le même temps, une étude récente (Chung et al., 2015 [42]) montre que même si les enseignants manifestent généralement une attitude positive envers les élèves avec TSA, cette attitude est significativement plus négative qu'envers les élèves tout-venants. De plus, cette recherche montre que les enseignants ayant une formation à l'éducation spécialisée ont une attitude significativement plus positive envers les élèves avec ASD que les enseignants sans formation à l'éducation spécialisée. Les enseignants de sexe féminin, et ceux travaillant dans le premier degré sont plus susceptibles d'avoir une attitude positive envers les élèves avec TSA que leurs collègues du sexe masculin et ceux travaillant dans le second degré. Dans l'ensemble, ces études mettent l'accent sur les besoins en termes de formation aux TSA des enseignants mais aussi de l'ensemble des personnels du système éducatif, ainsi que la nécessaire clarification du principe même d'inclusion (Segall \& Campbell, 2012 [47]).

\section{Nos choix méthodologiques}

L'objectif de cet article est, rappelons-le, de présenter les choix méthodologiques ayant guidés notre recueil de données auprès d'adolescents avec troubles du spectre autistique (TSA) en collège (en classe ordinaire et dans le dispositif Ulis). L'étude menée vise à mieux comprendre la nature et le déroulement des interactions sociales en classe entre un collégien avec TSA et son enseignant. Dans 
cette partie, nous décrivons d'abord les étapes préliminaires qui nous ont permis d'aboutir au recueil d'un corpus de données, puis nous présentons les méthodes de recueil de données utilisées et les raisons qui nous ont poussées à les adopter.

\subsection{Les étapes}

Plusieurs étapes préparatoires ont été nécessaires avant le début du recueil de données dans les collèges. Il a notamment fallu (1) constituer l'échantillon, c'est-à-dire déterminer la partie de la population concernée sur laquelle notre étude allait porter, (2) recueillir le consentement de tous les participants, (3) choisir et préparer les méthodes de recueil de données et (4) vérifier la conformité de notre projet avec la loi relative à l'informatique, aux fichiers et aux libertés ${ }^{\mathrm{d}}$. Dans cette partie, nous nous intéressons aux étapes 1, 2 et 4 ; les questions liées au choix des méthodes (étape 3) seront abordées dans la partie suivante (4.2).

\subsubsection{La constitution de l'échantillon}

La première étape dans la constitution de notre échantillon a été le recueil de l'autorisation des responsables de l'Education nationale: Directeurs académiques des services de l'éducation nationale (Dasen) et Inspecteurs de l'éducation nationale (IEN), pour mener notre recherche dans la zone géographique dont ils sont responsables.

Une fois les autorisations recueillies, la seconde étape a consisté à localiser les élèves avec TSA dans les établissements situés dans la zone géographique en question. Nous avons procédé comme suit

- nous avons d'abord demandé aux responsables de l'Education nationale (Dasen, IEN) les listes d'élèves avec TSA scolarisés en Ulis collège dans leur zone géographique,

- puis, nous avons contacté les chefs d'établissement (principaux de collège),

- ensuite, nous avons contacté les services du secteur médico-social spécialisé dans les TSA qui travaillent avec les établissements scolaires comme les SESSAD (Service éducatif de soin et de service à domicile),

- et enfin, nous avons activé notre réseau professionnel d'enseignant.

C'est la prise de contact directe avec les chefs d'établissement qui s'est s'avérée la plus efficace dans le cadre de notre étude : il s'agissait alors de contacter un à un les principaux de collège disposant d'une Ulis (sur une zone géographique déterminée) à partir d'une liste disponible sur internet et de leur demander si des élèves avec TSA étaient scolarisés dans l'établissement. Notre présentation en tant membre de l'Education nationale (en tant qu'ancienne enseignante d'Ulis spécialisée dans les TSA) a été un atout majeur pour engager le dialogue avec les professionnels et gagner progressivement leur confiance.

Ensuite, dans un troisième temps, nous avons pris contact avec les enseignants d'Ulis collège pour lesquels nous avions recueillis l'accord de principe du chef d'établissement. Lorsque ceux-ci étaient d'accord de participer à notre étude, nous avons évalué avec eux la faisabilité de notre démarche dans leur établissement. Ces enseignants, ayant pour fonction de coordonner la scolarité des élèves d'Ulis, connaissent particulièrement bien les élèves de l'Ulis, leur famille, les enseignants des classes ordinaires et les accompagnants. Ainsi, ils nous ont aidées à entrer en relation avec les enseignants des classes ordinaires, les jeunes avec TSA et leurs parents.

\footnotetext{
d Si les quatre étapes sont communes à toute recherche portant sur des élèves, le fait que les élèves auxquels nous nous intéressons soient en situation de handicap, demande un contrôle spécifique des données recueillies et de leur traitement par la Cnil.
} 


\subsubsection{Le recueil des consentements}

Une fois que les responsables de l'Education nationale ont donné leur autorisation et tous les participants leur accord pour participer à notre étude, nous avons procédé à l'établissement d'une convention de partenariat entre les établissements scolaires concernés et notre unité de recherche. Cette convention formalisait le déroulement de la recherche dans chaque établissement scolaire et les engagements des deux parties impliquées. Nous avons ensuite procédé au recueil du consentement ${ }^{\mathrm{e}}$ écrit des participants (enseignants, accompagnants, élèves et parents).

Le recueil du consentement auprès des participants s'est fait en deux temps : d'abord sous forme de rencontres et/ou de discussions puis sous forme de signatures des formulaires de demande de consentement et de demande de droit à l'image (ces derniers concernent les enseignants, les accompagnants et les élèves car nous avons choisi de les filmer en classe, cf. chapitre 4.2.2). Pour ce qui concerne les enseignants, des rencontres ont d'abord été organisées pour échanger sur notre travail et nos objectifs de recherche. Ensuite, des observations «blanches » (sans caméra) ont été mises en place afin de permettre aux enseignants d'envisager concrètement la présence d'un observateur extérieur dans leur classe. Ces observations blanches ont également été l'occasion pour nous de rencontrer les élèves (avec et sans TSA) et de leur expliquer nos objectifs de recherche. Ils ont ainsi pu poser des questions et dans certaines classes, cela a été l'occasion d'engager une réflexion sur l'inclusion des élèves avec TSA. Enfin, en ce qui concerne les parents des élèves avec TSA, nous les avons contactées directement afin de leur présenter notre projet et de leur expliquer leur rôle dans notre protocole de recherche. Enfin, après ces différentes rencontres et/ou discussions, les formulaires de demandes de consentement et de droit à l'image, accompagnés de documents explicatifs ont été transmis aux enseignants, aux accompagnants et à tous les élèves des classes (Ulis et ordinaire) ${ }^{\mathrm{f}}$ qui devaient les transmettre à leurs parents ; nous avons récupéré les formulaires complétés et signés dans les classes avant de commencer nos observations.

\subsubsection{La loi relative à l'informatique, aux fichiers et aux libertésg}

Nous avons également pris contact avec le correspondant Cnil de notre université de rattachement qui nous a d'abord informées des mesures juridiques à prendre dans la recherche au sens large et plus précisément pour la recherche dans le champ du handicap. Effet, lorsque l'intérêt d'un chercheur porte sur une population pour son handicap, il travaille à partir de données relatives à l'état de santé de cette population et de ce fait à partir de données dites « sensibles », soumises au secret médical. Ainsi, quel que soit le lieu du recueil de données (milieu scolaire, par exemple), la recherche peut rentrer dans le domaine de la recherche biomédicale et nécessiter une demande d'autorisation auprès de la Cnil pour la vérification de sa conformité avec la loi relative à l'informatique, aux fichiers et aux libertés. Après une analyse approfondie de tous les aspects (problématique, étapes, méthodes de recueil, de sauvegarde et d'exploitation des données) de notre projet de recherche, notre correspondant Cnil a estimé que notre recherche ne nécessitait pas de demande d'autorisation auprès de la Cnil. Dans ces conditions, il a procédé à l'inscription de notre recherche (et de tous les aspects mentionnés ci-dessus) à son registre de données. ${ }^{\mathrm{h}}$

\footnotetext{
${ }^{\mathrm{e}}$ Demande de consentement libre et éclairé et demande de droit à l'image.

${ }^{\mathrm{f}}$ Les formulaires et les documents explicatifs ont été transmis aux parents de tous les élèves des classes concernées (Ulis, classe ordinaire). En effet, même si notre étude concerne l'élève avec TSA, les autres élèves présents dans la classe étaient susceptibles d'apparaitre dans les vidéos réalisées. Nous avons reçu quelques refus des familles des camarades de classe des élèves avec TSA d'être filmés dans les classes ordinaires et en Ulis. Dans ces cas, nous avons soit fait en sorte de ne pas filmer l'élève concerné (lorsqu'un seul élève était concerné) soit nous avons exclu la classe en question de notre échantillon (lorsqu'il y avait plusieurs refus).

${ }^{g}$ Loi $\mathbf{n}^{\circ}$ 78-17 du 6 janvier 1978 relative à l'informatique, aux fichiers et aux libertés.

h Si le correspondant Cnil estime qu'une demande d'autorisation est nécessaire, le chercheur peut déposer directement sa demande auprès de la Cnil ou demander l'avis du Comité consultatif sur le traitement de l'information en matière de recherche dans le domaine de la santé (CCTIRS). Ce dernier l'aidera à préparer sa demande d'autorisation dans le respect des normes juridiques en vigueur.
} 


\subsection{Les méthodes}

Dans cette partie, nous présentons d'abord les méthodes de recueil de données utilisées dans notre étude et les raisons qui nous ont poussées à les choisir, puis le déroulement de ce recueil de données.

\subsubsection{Les méthodes choisies}

Comme nous l'avons vu précédemment (cf. chapitre 3.2), des études se sont intéressées aux interactions sociales entre les élèves avec TSA et leurs pairs en classe. De même, des études se sont intéressées aux interactions entre les élèves avec TSA et leur accompagnant (par exemple, Paquet et al., 2009 [48]). Dans ces recherches, c'est la méthode d'observation directe en classe qui a été privilégiée mais, la taille des échantillons est très réduite (de 3 à 6 participants). En ce qui concerne les recherches portant sur les enseignants des élèves avec TSA (cf. chapitre 3.5), elles se sont basées essentiellement sur le recueil de données par questionnaire. Les échantillons sont plus importants (de 53 à 234 participants selon les études) mais, il existe certaines limites connues à ce type de recueil de données. Par exemple, les déclarations recueillies ne corrèlent pas nécessairement avec les comportements réellement observés sur le terrain (Segall \& Campbell, 2012 [47]). De plus, la formulation des questions et les choix multiples proposés peuvent induire des réponses auxquelles les personnes n'auraient pas pensé spontanément. Il est également possible que seules les personnes les plus motivées remplissent et retournent le questionnaire ; cela pourrait entraîner un biais systématique dans les résultats (Segall \& Campbell, 2012 [47]).

Afin d'éviter ces biais, et dans le but d'obtenir une image la plus complète possible de la nature et du déroulement des interactions sociales en classe entre l'élève avec TSA et l'enseignant, nous avons fait le choix d'utiliser plusieurs méthodes de recueil de données sur un échantillon d'une douzaine d'élèves, leurs enseignants et leurs parents. Plus précisément, nous nous sommes inspirées du processus à quatre étapes proposé par Koegel et al. (2012 [41]) pour évaluer les situations scolaires impliquant des élèves avec TSA avant la mise en place d'une intervention pédagogique et éducative spécifique dans le cadre de l'inclusion. Selon ces auteurs, l'omission d'une ou de plusieurs des sources d'information suivantes est susceptible d'augmenter le risque de conclusions erronées :

- examen des dossiers des élèves,

- interview des professionnels travaillant avec les élèves,

- observation des élèves en milieu naturel,

- évaluation des élèves à partir d'un test standardisé.

Cette proposition est concordante avec celles d'autres chercheurs ayant suggéré de compléter les études avec questionnaires par l'observation directe des situations scolaires impliquant des élèves avec TSA (Roberston et al., 2003 [45]; Segall \& Campbell, 2012 [47]). De plus, elle va dans le sens de Loyd (2015 [49]) qui propose la prise en compte de la voix des personnes directement impliquées par la problématique de l'inclusion scolaire : les élèves avec TSA eux-mêmes.

Concrètement, nous avons commencé par observer en milieu naturel (c'est-à-dire en classe) les interactions de l'élève avec TSA et son enseignant à l'aide de la prise de vidéos (Bakeman \& Gottman, 2006 ; Pléty, 1993 ; Sinclair \& Coulthard, 1975 [50-52]). Dans un second temps, nous avons interviewé les différents partenaires impliqués dans l'interaction (enseignants, élèves) et rencontré les parents des élèves observés afin de récolter des informations sur les dossiers des élèves (leur diagnostic, leur parcours scolaire) ainsi que de faire compléter aux parents un test standardisé.

\subsubsection{Le déroulement du recueil de données}

Pour mettre en place l'observation en classe, nous avons suivi chaque élève avec TSA en Ulis avec l'enseignant spécialisé et, en classe ordinaire avec l'enseignant ordinaire. Nous avons utilisé deux caméras : une caméra fixe dotée un micro-cravate filmant l'élève avec TSA et une caméra mobile suivant l'enseignant. L'observation filmée a été reproduite trois fois pour chaque contexte (trois cours 
en Ulis et trois cours en classe ordinaire) et au total, nous avons recueilli environ six heures de données audiovisuelles pour chaque élève (un cours durant 55 minutes environ). La répétition des séances filmées avait pour objectif d'habituer les personnes observées à la présence du chercheur, à celle des caméras et de recueillir des données au plus près des situations réelles. L'intervalle de temps entre chacune de nos venues a été limité autant que possible afin de ne pas perdre l'effet d'habituation des participants au dispositif du chercheur. Cependant, l'organisation de nos venues s'est surtout faite en fonction de l'emploi du temps du jeune avec TSA (qui est assez complexe) et des événements de l'établissement (sorties scolaires, absence des enseignants, des élèves, etc.).

Pour mettre en place le recueil des propos des partenaires impliqués dans l'interaction, nous avons choisi de mener des entretiens avec les enseignants et les élèves. Nous avons choisi de les réaliser après les observations en classe afin de ne pas influencer le comportement de personnes filmées (par le biais des questions que nous souhaitions poser). En ce qui concerne plus particulièrement le recueil des propos des enseignants, nous avons mené des entretiens semi-directifs enregistrés. Nous souhaitions laisser un espace de parole ouvert aux enseignants afin qu'ils puissent s'exprimer sur leur parcours professionnel, leur rapport à l'inclusion scolaire, au handicap et aux TSA. Nous avons construit notre grille d'entretien sur la base des études menées sur les attitudes des enseignants vis-àvis des élèves avec TSA (McGregor \& Campbell, 2001 [44]). En ce qui concerne le recueil des propos des élèves avec TSA, nous nous sommes basées sur les recherches sur la cognition des élèves avec TSA et les pratiques d'adaptations pédagogiques (par exemple, Jordan \& Powell, 1997 [34]). Ainsi, au lieu de poser des questions ouvertes sur des sujets généraux, nous avons choisi de mener des entretiens directifs à l'aide d'une série de questions évoquant des exemples de situations concrètes pour lesquelles l'élève pouvait choisir une (ou des) réponse(s) proposée(s) et/ou proposer sa propre réponse. Quant au support de l'entretien, nous avons proposé un support écrit à l'élève et lui avons demandé s'il souhaitait lire les questions ou s'il préférait que nous les lui lisions car les apprenants avec TSA ont des difficultés à traiter les informations transmises oralement avec un débit verbal normal (Gepner \& Mestre, 2012 [53]). Enfin, nous avons utilisé des phrases interrogatives simples et avons exclu les expressions imagées et implicites car les élèves avec TSA ont des difficultés à comprendre les expressions complexes (Jordan et Powell, 1997 [34]).

Enfin, pour recueillir des informations complémentaires sur le dossier du jeune observé et administrer un test standardisé, nous avons rencontré les parents des jeunes observés. La nécessité de prendre connaissance du diagnostic exact de l'élève vient du fait que les TSA regroupent différents troubles (les troubles autistiques, le syndrome d'Asperger, le syndrome désintégratif de l'enfance et les troubles envahissants du développement non spécifiés) et que les classifications des troubles utilisées dans les centres de diagnostic en France peuvent être différentes d'un établissement à un autre (CIM-10, DSM-5 $5^{\mathrm{i}}$; de plus, les classifications évoluent dans le temps (cf. par exemple le passage du DSM-IV-TR au DSM-5 en 2013), ce qui multiplie les termes utilisés pour parler des élèves avec TSA. Quant au test standardisé, nous avons utilisé la version française de l'échelle de la Vineland II (Sparrow et al., 2015 [54]). Barnhill (2002 [55]) la définit de méthode indirecte d'évaluation des comportements sociaux et émotionnels en comparaison aux méthodes directes et expérimentales. Sa vocation est d'améliorer la compréhension des caractéristiques personnelles et sociales de l'élève avec TSA plutôt que d'évaluer les fonctions des comportements. Nous avons fait ce choix à partir de nos préoccupations de recherche portant sur les interactions sociales et des outils disponibles et validés en France.

Enfin, les grilles d'entretien ainsi que la Vineland II ont été pré testées auprès d'un enseignant d'Ulis qui travaille avec des élèves avec TSA, d'un enseignant de classe ordinaire qui accueille des élèves avec TSA dans sa classe, d'un élève avec TSA et sa famille qui ne participent par directement à la recherche. Ces essais nous ont permis de nous perfectionner dans la passation des entretiens et du test, de vérifier et réajuster notre protocole de recueil de données.

\footnotetext{
i CIM-10: Classification statistique internationale des maladies et des problèmes de santé connexes (10 ème révision). DSM-5 : Diagnostic and statistical manual of mental disorders $\left(5^{\text {th }} \mathrm{ed}\right.$.).
} 


\section{Conclusion}

Comme nous l'avons vu, les élèves avec TSA ont un fonctionnement cognitif et social bien particulier qui nécessite des interventions pédagogiques et éducatives spécifiques (Iavanonne et al., 2003 [56]; Jordan \& Powel, 1997 [34]). Leur présence à l'école ordinaire interroge et met en tension les enseignants qui les accueillent dans leur classe (Eman \& Farrell, 2009 [57]) ; ils sont d'ailleurs considérés comme les plus difficiles à inclure en classe ordinaire parmi les élèves à besoins éducatifs particuliers (House of Commons Education and Skills Committee, 2006 [58]). Dans le même temps, les enseignants ayant la responsabilité d'enseigner à ces élèves en milieu ordinaire disent manquer de connaissances et de formation sur les TSA (Humphrey \& Symes, 2013 [43]; Segall \& Campbell, 2012 [47]) et des études montrent qu'ils sont plus vulnérables au burnout que leurs collègues n'ayant pas cette responsabilité (Jennett et al., 2003 [58]). Pour autant, peu de recherches se sont intéressées aux interactions sociales se déroulant directement entre ces élèves et leurs enseignants. Nous considérons que l'analyse de ces interactions constitue un moyen privilégié de compréhension des phénomènes scolaires d'apprentissage, d'éducation et plus généralement d'intégration sociale et culturelle (Coulon, 1993 [1]), susceptible de faire évoluer la situation.

Dans cet article, notre objectif était de présenter le dispositif de recueil de données que nous avons mis en place pour étudier les interactions en classe entre un collégien avec TSA et son enseignant. Nous avons considéré que la description à la fois du contexte juridique et des recherches existantes portant sur ces jeunes et leur scolarisation était un préalable indispensable à la compréhension des choix méthodologiques réalisés. Compte tenu de ces éléments, et après une analyse des méthodes de recueil de données utilisées par d'autres chercheurs de ce domaine, nous avons présenté les quatre sources d'information auxquelles nous avons fait appel : observation des interactions élève-enseignant en milieu naturel, interview des enseignants et des élèves, examen des dossiers (TSA, scolaire) des élèves et évaluation des élèves à partir d'un test standardisé (cf. Koegel et al., 2012 [41]). Les différentes méthodes (observation filmée, entretien semi-directif et directif, test standardisé), même si elles forment le noyau dur autour duquel notre recueil de données s'est construit, ne constituent cependant qu'un élément parmi d'autres dans le dispositif méthodologique mis en œuvre : chaque étape s'est révélée indispensable. En effet, sans les nombreuses prises de contact préalables avec notre terrain de recherche (responsables du système éducatif, professionnels de terrain, élèves, familles, etc.) pour la constitution de l'échantillon et le recueil des consentements, et la mise en conformité de notre protocole avec la loi relative à l'informatique, aux fichiers et aux libertés, les données recueillies n'auraient pas la même portée.

\section{Bibliographie}

1. A. Coulon, Ethnométhodologie et éducation (PUF, Paris, 1993)

2. Secrétariat général pour la modernisation de l'action publique, Rapport final sur la scolarisation des élèves en situation de handicap (Ministère de l'Education nationale, 2013)

3. Direction générale de l'enseignement scolaire, Enquêtes 3 et 12 en milieu ordinaire (DGESCO, 2012)

4. American Psychiatric Association, Diagnostic and statistical manual of mental disorders (5ième éd.) (APA, Washington DC, 2013)

5. M.-H. Plumet, L'autisme de l'enfant, un développement sociocognitif différent (Armand Colin, Paris, 2014)

6. G. Dawson, D. Hill, L. Galpert, A. Spencer, L. Watson, J. Abnorm Child Psychol., 18, 335-345 (1990)

7. A.M. Wetherby, C.A. Prutting, J. Speech Hearing Res., 27, 364-77 (1984)

8. S. Feldstein, M. Konstantereas, J. Oxman, C.D. Webster, J. Communication Disorders, 15, 415460 (1982)

9. J.S. Bruner, Le développement de l'enfant : savoir faire, savoir dire (PUF, Paris, 1983) 
10. Y. Bruinsma, R.L. Koegel, L.K. Koegel, Ment. Retard. Dev. Disabil. Res. Rev., 10, 169-175 (2004)

11. A.N. Meltzoff, M.K. Moore, Science, 198, 75-78 (1977)

12. J. Piaget, La formation du symbole chez l'enfant (Delachaux \& Niestlé, Neuchâtel, 1945)

13. J. Nadel, Imitation et communication entre jeunes enfants (PUF, Paris, 1986)

14. J. Nadel, Imiter pour grandir : développement du bébé et de l'enfant avec autisme (Dunod, Paris, 2011)

15. S. Baron-Cohen, A.-M. Leslie, U. Frith, Cognition, 21, 37-46 (1985)

16. P. Vermeulen, Autisme et émotions, (De Boeck, Bruxelles, 2009)

17. T. Grandin, Ma vie d'autiste (Odile Jacob, Paris, 1992)

18. E. Schopler, G.B. Mesibov, High-functioning individuals with autism (Plenum Press, New York, 1992)

19. N. Poirier, C. Des Rivières-Pigeon, Le trouble du spectre de l'autisme : état des connaissances (Presses de l'Université du Québec, Québec, 2013)

20. L. Watkins, M. O’Reilly, M. Kuhn, C. Gevarter, G.E. Lanciono, J. Sigafoos, R. Lang., J. Autism Dev. Disorders, 45, 1070-1083 (2014)

21. L. Vandromme, Rev. Int. Psychol. Soc., 1, 57-74 (1993)

22. C. Berzin, C. Lebert-Candat, Psychologie et Education, 2, 9-13 (2006)

23. C. Tardif in J.-C. Grubard, M. Martinet, J.-P. Müh, B. Roge, Autisme et intégration (Presses Universitaires de Lille, Lille, 1994)

24. L. Kanner, Nervous Child, 2, 217-250 (1943)

25. G. Marcaggi, L. Bon, F. Eustache, B. Guillery-Girard, Revue de Neuropsychologie, 4, 310-319 (2010)

26. U. Frith, F. Happé, Cognition, 50, 115-132 (1994)

27. L. Mottron, L'autisme, une autre intelligence (Mardaga, Sprimont, 2004)

28. M.A. Preissler, Autism, 3, 231-248 (2008)

29. D. Tammet, Je suis né un jour bleu (Les Arènes, Paris, 2007)

30. S. Ozonoff, R.E. McEvoy, Dev. Psychopath., 6, 415-431(1994)

31. E. Schopler, G.B. Mesibov, Social behavior in autism (Plenum, New York, 1986)

32. R. Leaf, J.M. Eachin, Autisme et ABA : une pédagogie du progrès (Pearson Education France, Paris, 2006)

33. A. Beaugerie-Perrot, G. Lelord, Intégration scolaire et autisme (PUF, Paris, 1991)

34. R. Jordan, S. Powell, Les enfants autistes : les comprendre, les intégrer à l'école (Masson, Paris, 1997)

35. T. Peeters, L'autisme. De la compréhension à l'intervention (Dunod, Paris, 2008)

36. L. Wing, Autistic children. A guide for parents and professionals (Brunner/Mazel, New York, 1985)

37. Haute Autorité de Santé, Recommandation de bonne pratique. Autisme et autres troubles envahissements du développement : interventions éducatives et thérapeutiques coordonnées chez l'enfant et l'adolescent (HAS, Paris, 2012)

38. F. Corneau, J. Dion, J. Juneau, J. Bouchard, J. Hains, Revue de Psychoéducation, 43, 1-36 (2014)

39. K. Crosland, G. Dunlap, Beh. Modif., 36, 251-269 (2012)

40. J.K. Harrower, G. Dunlap, Beh. Modif., 25, 762-784 (2001)

41. L. Koegel, R. Matos-Freden, L. Russell, R. Koegel, Cog. Behav. Pract., 19, 401-412 (2012)

42. W. Chung, S. Chung, S. Edgar-Smith, R.B. Palmer, D. DeLambo, W. Huang, Current Issues Educ., 18, 2 (2015)

43. N. Humphrey, W. Symes, Int. J. Inclusive Educ., 17, 32-46 (2013)

44. E.M. McGregor, E. Campbell, Autism, 5, 189-207 (2001)

45. K. Robertson, B. Chamberlain, C. Kasari, J. Autism Dev. Disorders, 33, 123-130 (2003)

46. R. Soto, J.A. Pooley, L. Cohen, M. Taylor, Austr. J. Spec. Educ., 36, 97-111 (2012)

47. M.J. Segall, J.M. Campbell, Res. Autism Spectrum Disorders, 6, 1156-1167 (2012)

48. A. Paquet, J. Forget, N. Giroux, Canad. J. Educ., 32, 420-447 (2009) 
49. D. Loyd, Br. J. Learn. Disabilities, 43, 8-15 (2015)

50. R. Bakeman, J.M. Gottman, Observing interaction. An introduction to sequential analysis (Cambridge University Press, Cambridge, 2006)

51. R. Pléty, Ethologie des communications humaines. Aide-mémoire méthodologique (Presses universitaires de Lyon, Lyon, 1993)

52. J.McH. Sinclair, R.M. Coulthard, Towards an analysis of discourse. The English used by teachers and pupils (Oxford University Press, Oxford, 1975)

53. B. Gepner, D. Mestre, Trends Cog. Sci., 6, 455 (2002)

54. S.S. Sparrow, D.V. Cicchetti, D.A. Balla, Vineland-II : échelle d'évaluation du comportement socio adaptatif de Vineland (2 ${ }^{\text {ème }}$ éd.) (ECPA, Montreuil, 2015)

55. G.P. Barnhill, Assess. Effect. Intervent., 27, 47-55 (2002)

56. R. Iovannone, G. Dunlap, H. Huber, D. Kincaid, Focus Autism Other Dev. Disabil., 18, 150-165 (2003)

57. M.M. Emam, P. Farrell, Eur. J. Spec. Needs Educ., 24, 407-422 (2009)

58. House of Commons Education and Skills Committee, Special educational needs. Third report of session 2005-06 (The Stationary Office Limited, London, 2006)

59. H.K. Jennett, S.L. Harris, G.B. Mesibov, J. Autism Dev. Disorders, 33, 583-93 (2003) 
